\title{
Multiscale Fractal Analysis of Cortical Pyramidal Neurons
}

\author{
Andreas Schierwagen ${ }^{1}$, Luciano da Fontoura Costa $^{2}$, \\ Alán Alpár ${ }^{3}$, Ulrich Gärtner ${ }^{3}$ \\ ${ }^{1}$ Institute for Computer Science, University of Leipzig, D-04109 Leipzig, Germany \\ ${ }^{2}$ Institute of Physics at São Carlos, University of São Paulo, \\ 13560-970 São Carlos, SP, Brazil \\ ${ }^{3}$ Department of Neuroanatomy, Paul Flechsig Institut for Brain Research, \\ University of Leipzig, D-04109 Leipzig, Germany \\ Email: schierwa@informatik.uni-leipzig.de
}

\begin{abstract}
The present study used 3D data on neuronal morphology images to quantitatively characterize the phenotype of transgenic neurons. We calculated the multiscale fractal dimension (MFD) of reconstructed neuronal cells. It was shown that in a specific mouse mutant changes in the complexity of neuronal morphology correlate with changes in the MFD of dendrites of pyramidal neurons. Neurons in the mutant strain have lower peak fractal dimension compared with the wildtype, and a greater variety of the cell morphological phenotype.
\end{abstract}

\section{Introduction}

During brain development, neurons form complex dendritic and axonal arbors that reach a characteristic pattern and size. The development of arbor shape is partly determined by genetic factors and partly by interactions with the surrounding tissue. Important means for understanding gene function are provided by transgenic mice mutations. In these mutants, gene overexpression may affect several organs and tissues, including the brain. In a specific mouse mutant introduced in [1] a permanently active Ras protein in post-mitotic neurons is expressed in the primary somatosensory cortex resulting in a dramatically enlarged dendritic tree. In both cortical layers II/III and V, the total surface area and the total volume of dendritic trees is greatly increased. This is mainly caused by increased dendritic diameter and tree degree [2]. Topological complexity of pyramidal neurons in layers II/III, however, appeared hardly affected: Sholl analyses of both basal and apical dendrites revealed no differences between transgenic and wildtype mice regarding any parameters considered, i.e. numbers of intersections, branching points (nodes) and tips (leaves) [2]. These results suggest a rather proportional increase of dendritic tree size, without distinct changes in the space-filling properties.

The present study was intended to substantiate these findings by analyzing fractal aspects of dendritic tree shape. There are several methods for describing trees by fractal measurements (e.g. [3, 4]). Multiscale fractal analysis [5] seems 
Fig. 1. Retrogradely labelled pyramidal neurons in layers II/III of the primary somatosensory cortex (left), and a cell (transgenic neuron SE8, right) rendered with CVAPP
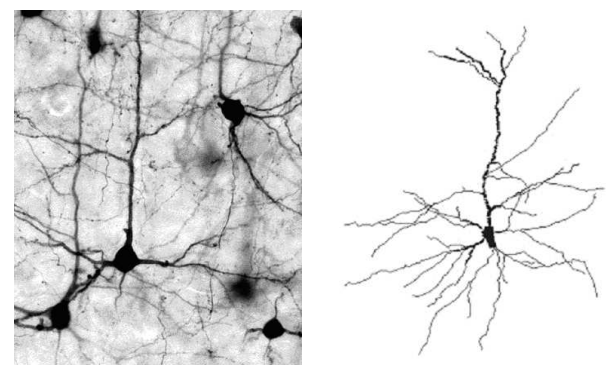

to be particularly suitable in the present case because the multiscale fractal dimension (MFD) is independent of size-related parameters like surface area and volume. The aim of this study is to show that observed changes in the complexity of neuronal morphology due to transgenic Ras activation in the primary somatosensory cortex of mice correlate with changes in the multiscale fractal dimensions of dendrites of pyramidal neurons.

\section{Materials and methods}

Neurons of two samples (17 pyramidal neurons of wildtype and 26 of transgenic type) were reconstructed and digitized using Neurolucida ${ }^{\mathrm{TM}}$, as described in [2]. The morphology files obtained were processed with CVAPP, a freely available program [6] for cell viewing, editing and format converting (Fig. 1).

After thresholding 3D binary images were obtained with the neuron shape represented by the set of 1-voxels. The binary neuron images were used to calculate the MFD, a measure related to image complexity [7]. It has been computed through the Minkowski sausages approach which can be described as follows:

Let the neuron shape under study be represented by the set $S$ of the Cartesian coordinates of each of its 1-voxels. Its exact dilation by a radius $r$ is defined as the union of all spheres of radius $r$ centered at each of the elements of $S$. A series of dilations on the image is made, with radii $r_{i}$ equivalent to the intrinsic lattice distances, the so-called exact distances. At each dilation, the volume $V\left(r_{i}\right)$ of the image is computed.

The volume $V(r)$ of the shape $S$ is therefore defined by

$$
V(r)=\sum_{i=1}^{M} V\left(r_{i}\right) \delta\left(r-r_{i}\right)
$$

where $\delta($.$) is the Dirac delta function and M$ is the index of the largest exact distance being considered. As $V(r)$ is a discontinuous function on $r$, which is a consequence of the discrete nature of $r_{i}$, it is necessary to interpolate between the 
Dirac deltas, which is here accomplished by convolving $V(r)$ with the Gaussian $g_{\sigma}(r)=1 / \sigma / \sqrt{2 \pi} \exp \left(-0.5(r / \sigma)^{2}\right)$ yielding the following interpolated volume

$$
v_{\sigma}(r)=\sum_{i=1}^{M} V\left(r_{i}\right) g_{\sigma}\left(r-r_{i}\right)
$$

It is important to choose a suitable value of the standard deviation parameter, $\sigma$, that is large enough just to interpolate between the largest gaps between the exact radii, which occur for small values of $r$. The cumulative volume is defined as

$$
C(s)=\int_{-\infty}^{s} v_{\sigma}(r) d r
$$

The Euclidean distance is now represented in terms of its logarithm, leading to the spatial scale parameter $s=\log (r)$, so that the exact radii are expressed as $s_{i}=\log \left(r_{i}\right)$. The multiscale fractal dimension $f(s)$ of the set $S$ of voxel elements then can be defined by

$$
f(s)=3-\frac{d}{d s} \log (C(s))=3-\frac{C^{\prime}(s)}{C(s)}
$$

While the traditional fractal dimension corresponds to a single scalar value, the MFD is a function of the spatial scale parameter $s$. Meaningful parameters of the MFD curve are: peak fractality, $f_{M}$, characteristic scale, $s_{M}$, and average fractality, $\langle f\rangle$. For the computational implementation of this method, see [4].

\section{Results}

In Figure 2, an example calculation of multiscale fractal dimension for the transgenic cell SE8 displayed in Fig. 1 is shown. Following the scheme defined in Eqns. 1-4, the multiscale fractal dimension depending on $s$ was obtained.

As shown in Fig. 2, the fractal dimension decreases at both micro and macro scales, and a peak fractal dimension value, $f_{M}$, is observed at an intermediate scale value, $s_{M}$. Another relevant parameter is the average fractal dimension, $<f>$.

The sample histograms of the three parameters utilized, $f_{M}, s_{M}$, and $<f>$, are presented in Fig. 3. For $f_{M}$, a two mode distribution for the transgenic cases results, while wildtype cells produced a single mode (Fig. 3a). The distribution of the spatial scales where the peak fractality $s_{M}$ is observed suggests that the two types of cells are characterized by similar values of this parameter (Fig. 3b). Finally, the distributions of $\langle f\rangle$ are bimodal in the case of transgenic cells, and unimodal for wildtype cells (Fig. 3c).

Fig. 4 shows the Gaussian densities after principal component analysis for the parameter combinations $\left(f_{M}, s_{M}\right)$ and $\left(f_{M},<f>\right)$, after normal statistical transformation (leading to null mean and unit variance in both cases). Obviously, 
Fig. 2. The multiscale fractal dimension of a neuron (cell SE8)

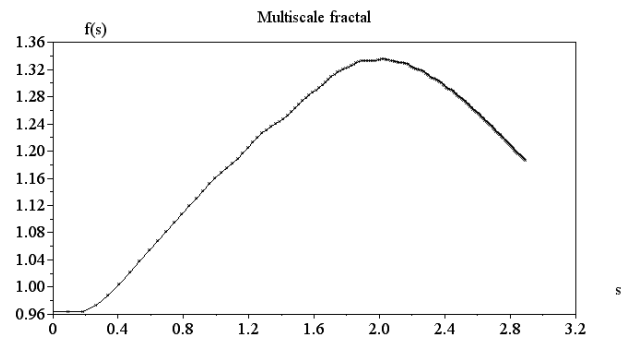

Fig. 3. Histograms: Peak fractal dimension $f_{M}$ (a), maximum fractality scale $s_{M}$ (b), average fractal dimension $\langle f\rangle$ (c). Wildtype and transgenic cases are identified by diamonds and crosses, resp.

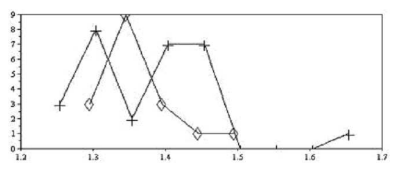

(a)

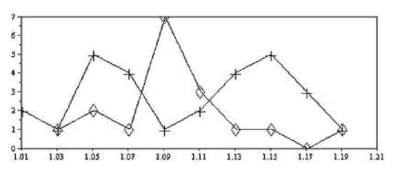

(b)

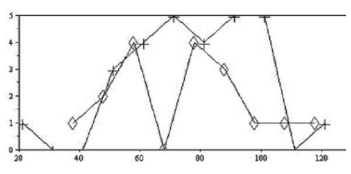

(c)

the parameter combination $\left(f_{M}, s_{M}\right)$ enables a better separation of the two cell types. As indicated in Fig. 4 (left), transgenic cells tend to be less complex (lower fractality), expressing at the same time greater variance.

\section{Discussion}

The present study is one of the few which used 3D data on neuronal morphology to quantitatively characterize the morphological phenotype of neurons. We calculated the multiscale fractal dimension of neuronal cells reconstructed in 3D. The advantages of the multiscale fractal dimension (a function of the spatial scale) over the traditional fractal dimension (a single scalar value) reside in providing additional information about the analyzed shapes. Thus, we calculated complementing parameters such as the peak fractality, the spatial scale where it occurs, and the average fractality, for quantifying and characterizing the cell types.

Two sets of neurons, i.e. pyramidal cells from wildtype and transgenic mice, have been analyzed. The results obtained after principal component analysis mark transgenic neurons as slightly less complex, if measured by the peak fractal dimension, $f_{M}$, compared to their wildtype counterpart, while the other two parameters considered (maximum fractality scale, $s_{M}$, and average fractal dimension, $\langle f>$ ) did not reveal differences between the two types. Transgenic pyramidal neurons are characterized by increased dispersion when compared to the wildtype pyramidal neurons, suggesting that the enhanced Ras activity in transgenic mice may lead to greater variety of the cell morphological phenotype. 
Fig. 4. Gaussian densities for $\left(f_{M}, s_{M}\right)$ (left) and $\left(f_{M},<f>\right)$ (right). Wildtype and transgenic cases are marked by diamonds and crosses, respectively
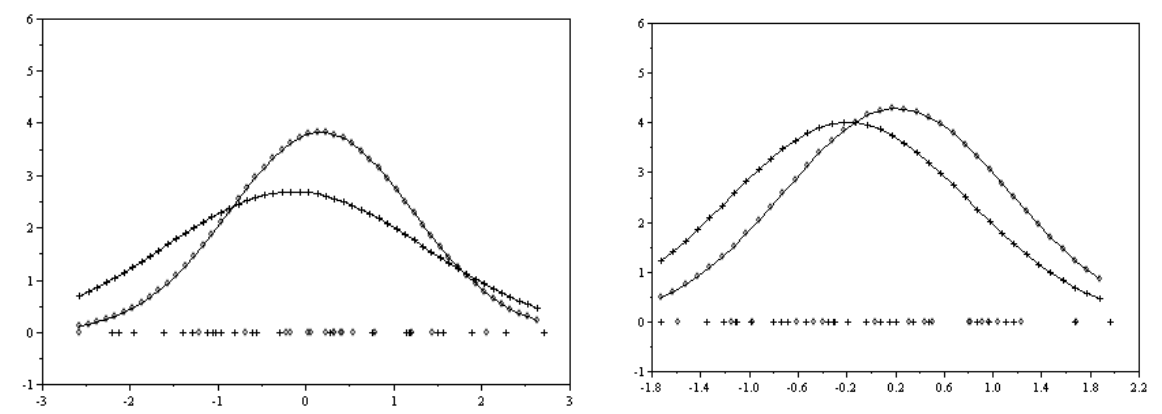

Neuronal shape analysis requires the specification of appropriate shape measures and corresponding computational methods. An important problem is the discriminative power of the particular shape measures. It is known that multiscale analyses tend to augment the resolution power of geometric descriptors [7]. In this context, our results suggest to consider the MFD as measure with discriminative strength. Only recently, we could confirm our findings with an alternative method, i.e. percolation analysis [8].

\section{References}

1. Heumann R, Goemans C, Bartsch D, et al. Constitutive activation of Ras in neurons promotes hypertrophy and protects from lesion-induced degeneration. J Cell Biol 2000;151:1537-1548.

2. Alpár A, Palm K, Schierwagen A, et al. Expression of constitutively active p21HrasVal12 in postmitotic pyramidal neurons results in increased dendritic size and complexity. J Comp Neurol 2003;467:119-133.

3. Schierwagen A. Dendritic branching patterns. In: Degn H et al. Chaos in Biological Systems. Plenum, New York; 1987. 191-193.

4. Fernandez E, Jelinek HF. Use of fractal theory in neuroscience: Methods, advantages, and potential problems. Methods 2001;24:309-321.

5. Costa LF, Manoel ETM, Faucereau F, et al. A shape analysis framework for neuromorphometry. Comput Neural Syst 2002;13:283-310.

6. Cannon RC. Structure editing and conversion with cvapp; 2000. Available from: http://www.compneuro.org/CDROM/nmorph/usage.html

7. Costa LF, Jr RCesar. Shape Analysis and Classification: Theory and Practice. CRC Press, Boca Raton; 2001.

8. Costa LF, Barbosa MS, Schierwagen A, et al. Active percolation analysis of pyramidal neurons of somatosensory cortex: A comparison of wildtype and p21H-rasVal12 transgenic mice. Int J Mod Phys C 2005;16:655-667. 